\title{
Erratum to: Multivariate comparison of B-ultrasound guided and laparoscopic continuous circulatory hyperthermic intraperitoneal perfusion chemotherapy for malignant ascites
}

Ming-Chen Ba $\cdot$ Hui Long $\cdot$ Shu-Zhong Cui $\cdot$

Yun-Qiang Tang • Yin-Bing Wu $\cdot$ Xiang-Liang Zhang •

Hong-Sheng Tang $\cdot$ Sai-Xi Bai

Published online: 24 January 2014

(C) Springer Science+Business Media New York 2014

Erratum to: Surg Endosc (2013) 27:2735-2743

DOI 10.1007/s00464-013-2800-3

The right data of the incidence of port-site metastasis in B ultrasound-guided group should be $9.375 \%$, in laparoscopically-assisted group should be $10 \%$, but the error data of our calculation in B ultrasound-guided group was $18.75 \%$, and in laparoscopically-assisted group was $18.18 \%$ in text and table of the published paper.

The online version of the original article can be found under doi:10.1007/s00464-013-2800-3.

M.-C. Ba (ه) · S.-Z. Cui · Y.-Q. Tang · Y.-B. Wu •

X.-L. Zhang $\cdot$ H.-S. Tang $\cdot$ S.-X. Bai

Intracelom Hyperthermic Perfusion Therapy Center,

Cancer Hospital of Guangzhou Medical College,

Guangzhou 510095, China

e-mail: bamingchen2011@126.com

H. Long $(\bowtie)$

Department of Pharmacy, Guangzhou Dermatology Institute,

Guangzhou 510095, China

e-mail: 798587414@qq.com 